\title{
Macrolide therapy in cryptogenic organizing pneumonia: A case report and literature review
}

\author{
QUN-LI DING, DAN LV, BI-JIONG WANG, QIAO-LI ZHANG, YI-MING YU, SHI-FANG SUN, \\ ZHONG-BO CHEN, HONG-YING MA and ZAI-CHUN DENG
}

Department of Respiratory Medicine, Affiliated Hospital, School of Medicine,

Ningbo University, Ningbo, Zhejiang 315020, P.R. China

Received May 9, 2014; Accepted December 15, 2014

DOI: $10.3892 / \mathrm{etm} .2015 .2183$

\begin{abstract}
Cryptogenic organizing pneumonia (COP) is a pulmonary disorder associated with nonspecific clinical presentations. The macrolide class of antimicrobial agents is widely used to treat infectious and inflammatory respiratory diseases in humans. The present study reports a case of COP that was effectively treated with azithromycin in combination with glucocorticoid. A literature review of similar cases is also presented. It was found that all COP patients in the literature received macrolide treatment, including six cases with unknown clinical outcomes. For the remaining 29 patients, 20 patients initially received the macrolide as a single therapy and $4 / 5$ of them (16 cases) were cured with a treatment time of 3-14 months, while 1/5 (4 cases) showed no improvement after treatment for 1 month and were switched to a glucocorticoid or combination treatment with a glucocorticoid, after which the disease was finally well-controlled. Side-effects of macrolide were rare. Based on this analysis, it is recommended that macrolides can be used as a first-line therapy in patients with mild COP. For patients with recurrent COP, it is suggested that macrolides should be used as an adjunctive therapy with other treatments, such as a glucocorticoid.
\end{abstract}

\section{Introduction}

Organizing pneumonia (OP) is a pulmonary disorder that is associated with nonspecific clinical presentations, chest radiographic findings and pulmonary function results (1). OP is divided into primary and secondary OP based on etiology. Primary OP is referred to as cryptogenic organizing pneumonia (COP) and classified as an idiopathic interstitial

Correspondence to: Professor Zai-Chun Deng, Department of Respiratory Medicine, Affiliated Hospital, School of Medicine, Ningbo University, 247 Renmin Road, Ningbo, Zhejiang 315020 , P.R. China

E-mail: dengzaichun982136@hotmail.com

Key words: macrolide, organizing pneumonia, treatment, inflammation, glucocorticoid, azithromycin pneumonia $(1,2)$. Secondary OP is associated with a number of entities, including drugs, infections, malignancies, connective tissue diseases, organ transplantation, radiotherapy and the inhalation of harmful gases. COP mainly involves the alveoli, alveolar ducts and small airways; however, the lung interstitium may also be involved. It is considered as an inflammatory disease and diagnosed based on the clinical, radiographic and pathological findings following the exclusion of diseases associated with secondary OP (3). Glucocorticoids are effective in the treatment of COP. However, glucocorticoids usually take a longer time to take effect, and this results in severe side-effects.

Macrolides, a class of antimicrobial agents first isolated from Streptomyces erythreus more than five decades ago, are widely used to treat infectious respiratory diseases in humans (4). In addition to the anti-infectious effect, macrolides have been reported to have anti-inflammatory effects on certain inflammatory respiratory diseases, including asthma, bronchiectasis and cystic fibrosis (CF) (4). Studies have shown low-dose macrolides to be efficient in the treatment of COP (5-7). However, those studies are limited to case-based reports. There is a lack of controlled comprehensive clinical trials and definitive recommendations. The present study reports a case of COP that was effectively treated with azithromycin in combination with a glucocorticoid. A search of the literature was carried out in Medline, using the keywords 'macrolide OR azithromycin OR erythromycin OR roxithromycin OR clarithromycin' AND ‘cryptogenic organizing pneumonitis OR bronchiolitis obliterating organizing pneumonia'. A total of 35 articles were retrieved by December 2012. After excluding unrelated and repetitive articles, eight papers were selected for analysis. A review of 35 cases of $\mathrm{OP} / \mathrm{COP} /$ bronchiolitis obliterans organizing pneumonia (BOOP) treated with macrolide antibiotics, from these papers and including the current case, was conducted.

\section{Case report}

A 58 year-old female patient was admitted to the Affiliated Hospital of Ningbo University School of Medicine (Ningbo, China) with a nine-month history of concurrent cough, intermittent mild fever and fatigue. Prior to coming to the hospital, the patient had been treated with cephalosporins, quinolones and macrolides in other hospitals and the symptoms had not 
improved. The patient had been hypertensive for more than 10 years and was taking nifedipine orally to maintain a normal blood pressure. Physical examination revealed crackles in both lungs. The examination was otherwise normal, with no signs of connective tissue disorders. Chest computed tomography (CT) scanning showed bilateral nodular, patchy alveolar opacities, prominently in the right lung, and thickened pleura (Fig. 1). Laboratory tests showed a white blood cell (WBC) count of $8.3 \times 10^{9} / 1$, a neutrophil percentage of $63 \%$, eosinophil percentage of $0.7 \%$, red blood cell count of $4.46 \times 10^{12} / 1$, hemoglobin concentration of $116 \mathrm{~g} / \mathrm{l}$, platelet count of $369 \times 10^{9} / 1$, erythrocyte sedimentation rate of $112 \mathrm{~mm} / \mathrm{h}$ and C-reactive protein level of $9.1 \mathrm{mg} / \mathrm{l}$. Hepatic and renal function were normal. The serum anti-mycoplasma antibody titer was 1:80. The anti-double stranded DNA titer was $136 \mu \mathrm{g} / \mathrm{l}$, the immunoglobulin E level was $41.58 \mathrm{kIU} / \mathrm{l}$ and the anti-paragonimiasis antibody test was negative. Immunological examinations were all normal. Perinuclear anti-neutrophil cytoplasmic antibody (P-ANCA) and cytoplasmic-ANCA tests were negative, and the tumor markers CA19-9, CA-125, carcinoembryonic antigen, neuron-specific enolase and CYFRA21-1 were normal. The blood gas $\mathrm{pH}$ was 7.41 , the partial pressure of $\mathrm{CO}_{2}$ in arterial blood $\left(\mathrm{PaCO}_{2}\right)$ was $39 \mathrm{mmHg}$, the $\mathrm{PaO}_{2}$ was $96 \mathrm{mmHg}$ and the arterial oxygen saturation $\left(\mathrm{SaO}_{2}\right)$ was 98\%. Sputum culture was negative. Pulmonary function tests indicated small airway dysfunction. Bronchoscopy revealed a normal tracheal mucosa and lumen. A test of bronchoalveolar lavage fluid (BALF) showed no exfoliated cells. The cell types were $2.4 \%$ neutrophils, $0 \%$ eosinophilic granulocytes, $82.3 \%$ macrophages and $15.3 \%$ lymphocytes. Transbronchial needle aspiration did not detect cancer cells. The patient was initially considered to have community acquired pneumonia and was treated with moxifloxacin ( $0.4 \mathrm{~g}$, intravenous drip, daily). Ten days later, a chest CT scan demonstrated that the lesions on the right upper lung were slightly absorbed; however, there were new nodular and patchy ground glass opacities in the left lung (Fig. 2). A percutaneous lung biopsy in the lower left lung was performed. The pathologic examination identified typical characteristics of OP. The alveoli and alveolar ducts were filled with plugs of granulation tissue composed of fibroblasts. Chronic inflammatory cell infiltration and a few scattered giant cells were found in these tissue sections (Fig. 3). The patient was treated with $0.75 \mathrm{mg} / \mathrm{kg} / \mathrm{day}$ of prednisone orally. Two weeks later, respiratory symptoms disappeared and chest radiographic abnormalities were improved. After another 2 weeks of prednisone treatment, the patient complained of gastrointestinal discomfort. Thus, $500 \mathrm{mg} /$ day of azithromycin was added to the treatment, and the dose of prednisone was gradually reduced. Three months later, prednisone was completely withdrawn. The patient was continually given azithromycin at $500 \mathrm{mg} / \mathrm{day}$ for another three months. A chest CT scan showed a complete resolution of the previous bilateral patchy, nodular consolidations in both lungs (Fig. 4). The patient was followed-up for one year, and the disease did not relapse. Informed consent was obtained from the patient.

\section{Discussion}

COP is a type of organizing pneumonia without the presence of evident pathogens, such as infection, or other associated

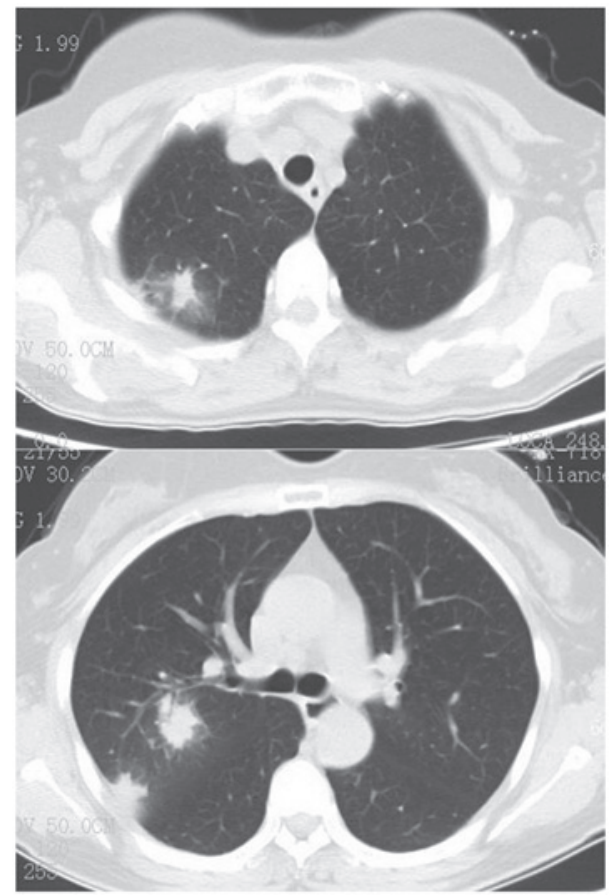

Figure 1. Initial chest radiography showing patchy, nodular consolidation in the right lung, and some localization in the subpleural area.

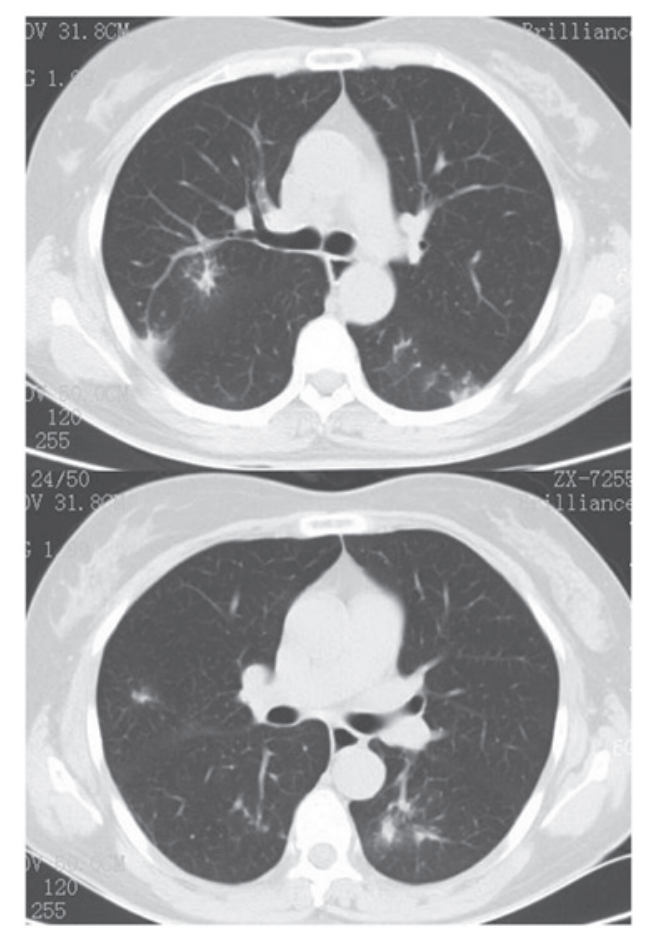

Figure 2. Chest radiography ten days after treatment showing patchy, nodular consolidation in the right lung and the lower lobe of the left lung.

diseases, such as connective tissue disease. COP is commonly observed in the non-smoking population and predominantly in female patients. In the present review of 35 cases of OP/COP/BOOP, there were 12 male and 23 female patients. Their ages were between 13 and 83 years. Radzikowska et al (6) reported that 10/12 patients were non-smokers, and 9/12 were female patients. In the cases covered in the current review, 


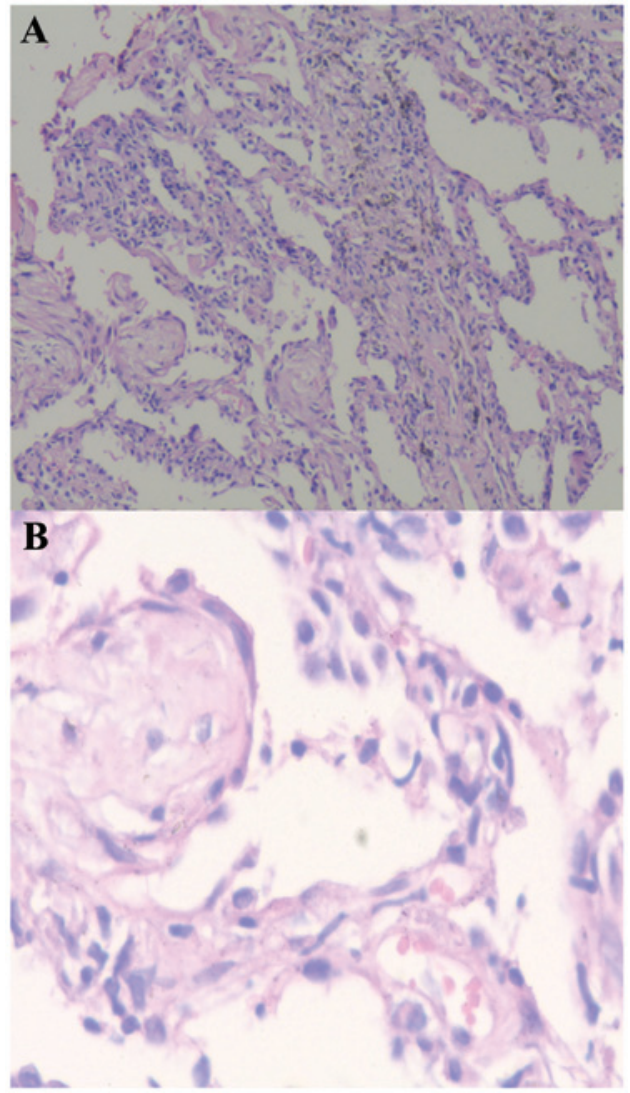

Figure 3. Histological changes in lung sections following lung biopsy. (A) Lower-power image showing patchy filling of the lung alveoli and respiratory bronchioles by loose plugs of granulation tissue (hematoxylin and eosin staining; magnification, x10). (B) Higher-power image showing the swirled intra-airway plugs of fibroblasts and inflammatory cells compatible with organizing pneumonia (hematoxylin and eosin staining; magnification, $\mathrm{x} 40$ ).

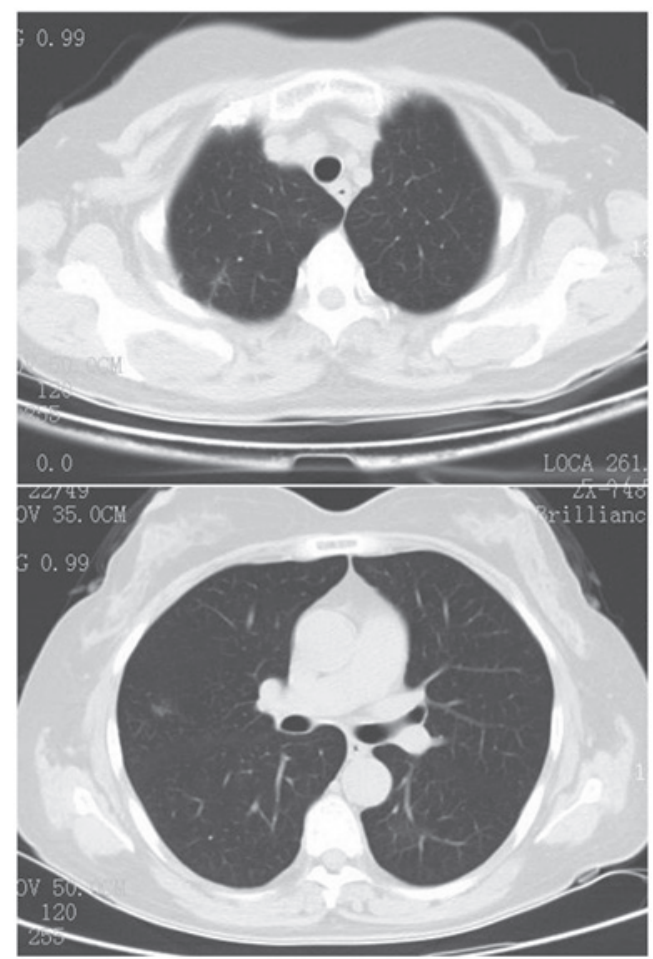

Figure 4. Chest radiography six months post-treatment showing a complete resolution of the previously observed bilateral patchy, nodular consolidations in the two lungs. there were two smokers, four ex-smokers and 14 non-smokers; the smoking history of the remaining nine patients was not mentioned in the papers. There were 23 cases of primary COP and six cases of secondary COP. Three cases were associated with radiotherapy, and the other three cases were associated with amiodarone, chemotherapy and bone marrow transplantation, respectively. None of them had family history of COP.

COP patients usually do not show unique clinical presentations. Among the 35 reviewed cases, 29 patients had certain types of symptoms; the symptoms of the other six patients were not specified. The most common symptoms were cough (24/29), shortness of breath (18/29), light fever (16/29), fatigue $(15 / 29)$, weight loss $(11 / 29)$ and night sweats $(6 / 29$; Table I). The complications included pneumothorax in one case (5), and mediastinal emphysema and pneumothorax in one case (4). The interval time from onset of symptoms to diagnosis was variable, with the longest being a 5-year medical history for a 60 -year-old female patient. During these 5 years, the patient repeatedly presented with fever, cough and shortness of breath. The patient was initially diagnosed with asthma, and was pathologically diagnosed with COP through percutaneous lung biopsy (7). Physical examinations were usually normal in these patients. Nine out of 29 patients had lung wheezing, and two had lung crackles. The majority of the patients had mild illnesses; only one patient had acute onset of illness, and the illness rapidly progressed following prednisone treatment, and had complications of pneumomediastinum and pneumothorax. The disease was improved after the patient was given macrolide in combination with methylprednisolone and cyclosporine (8).

The majority of the patients had abnormal chest radiographic findings. The most common manifestations were multiple patchy opacities (21/29), air bronchogram (16/29), multiple lung nodules (5/29), ground glass opacity $(5 / 29)$, multiple consolidation (2/29) and single consolidation (3/29; Table I). The most common feature in the chest radiography of patients with COP was a migratory pattern. It existed in 9/12 patients in a single study (75\%) (6). In the present review, it was observed in $37.9 \%$ of patients (11/29). The majority of the lesions involved bilateral lungs; both right and left lungs were involved in 28 cases. Pleural effusion and lymphadenopathy were rare. The radiographic findings were variable in OP, and the differentiation of OP from other pulmonary diseases is challenging. Lung biopsy is extremely important to the diagnosis of OP. All 35 patients were diagnosed by either cytology or pathology. Transbronchial lung biopsy (TBLB) was the most frequently used technique, and it had the highest diagnostic value among all methods. Thirteen out of 15 patients were diagnosed through TBLB. TBLB failed to diagnose the remaining two cases, which were diagnosed through thoracoscopy. Four cases were diagnosed through percutaneous lung biopsy, one of them was confirmed at the second examination. Seven patients were confirmed by surgery and three were confirmed by thoracoscopy. Surgical intervention is not recommended as the first choice due to its invasiveness. TBLB is strongly recommended as a routine diagnostic technique for COP patients.

COP is considered to be an inflammatory disease. BALF cytological examination demonstrated increased lymphocyte counts, decreased CD4/CD 8 ratio and increased $\mathrm{CD} 8{ }^{+} \mathrm{CD} 11 \mathrm{~b}$ cell levels (9). In this review, BALF cytological classification 
Table I. Clinical characteristics of patients with COP.

\begin{tabular}{ccc}
\hline No. of & $\begin{array}{c}\text { Percentage } \\
\text { of total }\end{array}$
\end{tabular}

\section{Gender}

Male

Female

Age

13-83 years

Smoking

$\begin{array}{lr}\text { Never } & 14 / 20 \\ \text { Ex-smoker } & 4 / 20 \\ \text { Smoker } & 2 / 20 \\ \text { COP } & \end{array}$

Primary

Secondary

Symptoms

$$
\text { Cough }
$$

Dyspnea

Low-grade fever

Weight loss

Fatigue

Night sweats

$4 / 20$
$4 / 20$

70

20

10

82.8

82.8

62.1

55.2

37.9

51.7

20.7

Lung signs

Crackles

Wheezing

Chest radiograph

Normal

Abnormal

Patchy consolidation

Bilateral

Multiple nodules

Pleural effusion

Migratory lesions

Ground glass opacities

Air bronchogram

2

9

6

29

72.4

96.6

17.2

6.9

37.9

17.2

55.2

Lung biopsy

TBLB

Thoracoscopy

Percutaneous lung biopsy

Surgery

Macrolide

$\begin{array}{lrr}\text { Alone } & 5 & 82.8 \\ \text { Adjunctive } & 24 & 17.2 \\ \text { Efficacy } & & \\ \text { Effective } & 24 & 82.8 \\ \text { Invalid } & 5 & 17.2\end{array}$

COP, cryptogenic organizing pneumonia; TBLB, transbronchial lung biopsy. ${ }^{a} n=29$ with known outcome; $n=20$ with known smoking status.

was dominated by lymphocytes $(8 / 16 ; 50 \%)$. Cai et al (10) observed that the cytokines interleukin (IL)-6, -8, and -10 , interferon- $\gamma$-inducible protein 10 , tumor necrosis factor (TNF)- $\alpha$, soluble TNF receptor 1 (STNFR1) and STNFR2 in BALF were significantly increased in COP patients, and macrolides significantly decreased their levels. The proportions of lymphocytes, neutrophils and $\mathrm{CD}^{+} \mathrm{CD}^{+} \mathrm{bb}^{-}$cells were significantly decreased following macrolide treatment (10). This suggests that increased lymphocyte counts and numbers

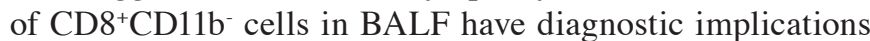
for COP.

Glucocorticoids are traditionally a first-line agent for treating COP. The majority of patients respond well to treatment (3). However, one study reported that $10-15 \%$ of patients were resistant to treatment and the disease progressed rapidly (11). A total of $13-58 \%$ of patients had recurrent disease during the reduction of glucocorticoids or following drug discontinuation (11). Macrolides, as regulators of immune responses, have been widely used in bronchial asthma, bronchiectasis, diffuse panbronchiolitis and other diseases. They have also been reported to effectively treat COP $(6-8,10,12-14)$. In the current review, all 35 patients received macrolide treatment, including six cases with unknown clinical efficacy. The efficacy was assessed in the remaining 29 patients. Twenty patients initially received macrolide as a single agent. Four-fifths of the patients (16 cases) were cured with the medication after 3-14 months; however, the improvement took a longer time than that of glucocorticoids. It usually took 2-3 weeks for symptom improvement and 1 month for radiological improvement on chest images. One-fifth of the patients (four cases) had no improvement following macrolide treatment for 1 month and had to switch to glucocorticoids or a combination treatment with glucocorticoids. After that, the disease was well-controlled. The most commonly used macrolide was clarithromycin (19 cases), the second was erythromycin (seven cases), and the next was azithromycin (two cases). The type of macrolide used was undocumented in one case. Nine patients were later treated with macrolide due to poor efficacy or side-effects from glucocorticoids. Following the addition of macrolide, the symptoms in these patients were significantly improved (Table II). There was one patient with severe COP in whom the disease continued to deteriorate during glucocorticoid treatment. Following treatment with a combination of cytotoxic drugs and macrolide, the disease attenuated rapidly (8). Side-effects of macrolide treatment were rare. There was only one patient who had a skin rash resulting from clarithromycin, and later this drug was stopped (14).

The molecular mechanism of macrolides in the treatment of COP is not fully understood. Pathologically, COP is mainly manifested as a polypoid granulation hyperplasia in the small airway and alveolar respiratory bronchioles. The granulation tissue is composed of fibroblasts/myofibroblasts, infiltrated with inflammatory cells, including monocytes, macrophages, mast cells, a few eosinophils and neutrophils, particularly in the early stage of the disease. Studies have shown that macrolides exhibit anti-inflammatory effects (15-20). They have been widely used in a variety of acute and chronic respiratory diseases and are effective in treating these diseases. Kudoh (21) suggested that erythromycin significantly improved the survival rate of patients with diffuse panbronchiolitis. Itkin and Menzel (22) demonstrated that macrolides could lower airway 


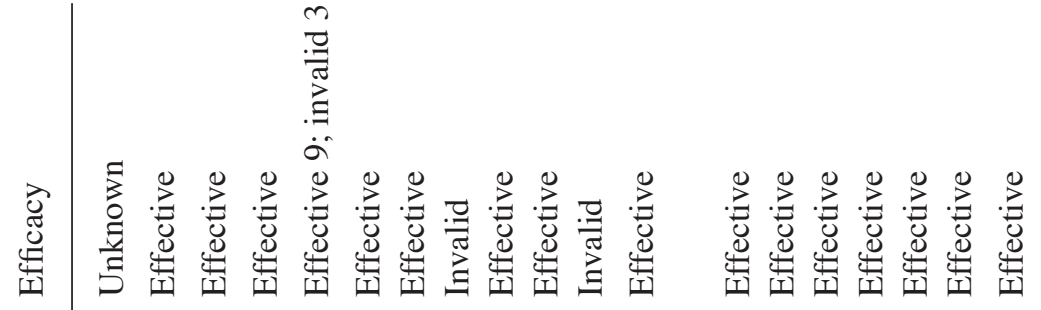

(1)

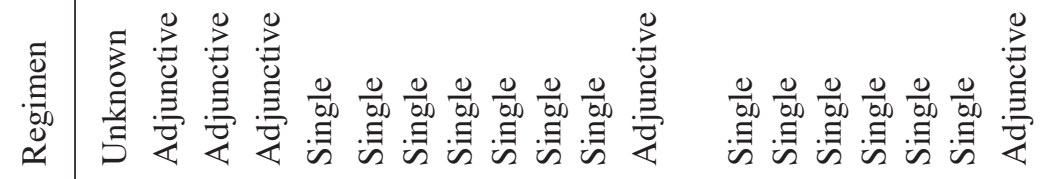

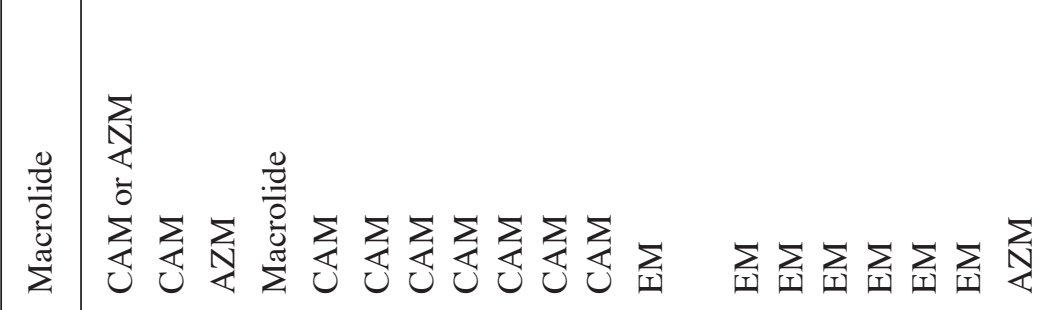

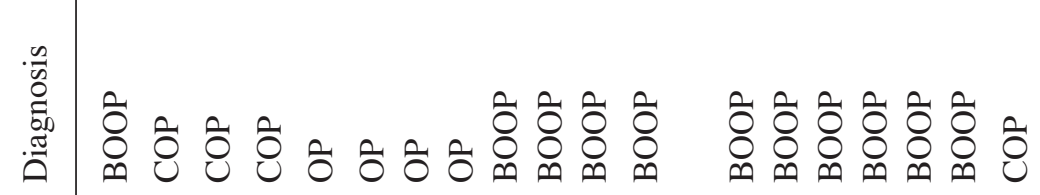

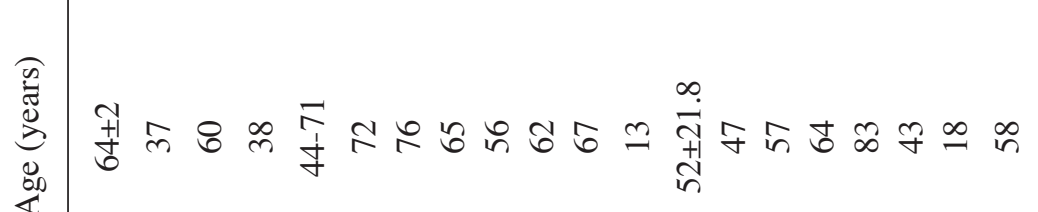

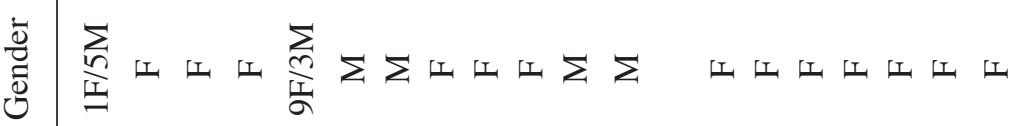

$0--20$

$-6$

$-$

ஓ ๙

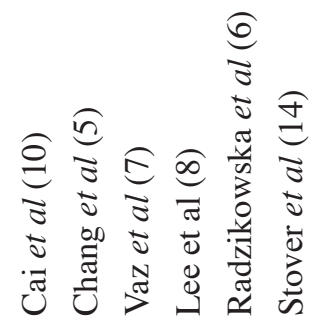

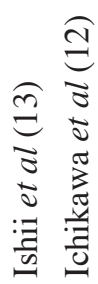

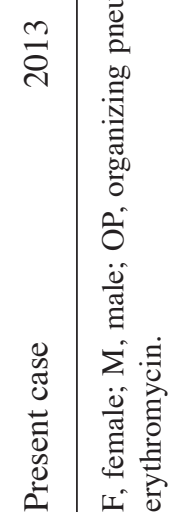


hyper-responsiveness and reduce the amount of corticosteroid used in patients with asthma. These lines of evidence support the anti-inflammatory effect of macrolides in the treatment of COP. The efficacy of a macrolide not only relies on its treatment time but also its molecular structure. The 14- and 15-membered ring antibiotics have been reported to have anti-inflammatory effects, and these antibiotics include macrolides such as erythromycin, clarithromycin and azithromycin (23).

Based on the present analysis, it is recommended that macrolides may be used as first-line therapeutic agents in patients with mild COP. For patients with recurrent COP or during the reduction of glucocorticoid dosing, it is suggested that macrolides may be combined with steroids as an adjuvant therapy. Prior to any definitive recommendations being made, large-scale randomized controlled clinical trials are required.

\section{References}

1. Drakopanagiotakis F,Paschalaki K,etal:Cryptogenic and secondary organizing pneumonia: clinical presentation, radiographic findings, treatment response and prognosis. Chest 139: 893-900, 2011.

2. American Thoracic Society and European Respiratory Society: American Thoracic Society/European Respiratory Society International Multidisciplinary Consensus Classification of the Idiopathic Interstitial Pneumonias. This joint statement of the American Thoracic Society (ATS) and the European Respiratory Society (ERS) was adopted by the ATS board of directors, June 2001 and by the ERS Executive Committee, June 2001. Am J Respir Crit Care Med 165: 277-304, 2002.

3. Drakopanagiotakis F, Polychronopoulos V and Judson MA: Organizing pneumonia. Am J Med Sci 335: 34-39, 2008.

4. Hatipoglu U and Rubinstein I: Low-dose, long-term macrolide therapy in asthma: An overview. Clin Mol Allergy 2: 4, 2004.

5. Chang WJ, Lee EJ, Lee SY, In KH, Kim CH, Kim HK and Park S: Successful salvage treatment of steroid-refractory bronchiolar COP with low-dose macrolide. Pathol Int 62: 144-148, 2012.

6. Radzikowska E, Wiatr E, Gawryluk D, et al: Organizing pneumonia - clarithromycin treatment. Pneumonol Alergol Pol 76: 334-339, 2008 (In Polish).

7. Vaz AP, Morais A, Melo N, et al: Azithromycin as an adjuvant therapy in cryptogenic organizing pneumonia. Rev Port Pneumol 17: 186-189, 2011 (In Portuguese).

8. Lee J, Cha SI, Park TI, Park JY, Jung TH and Kim CH: Adjunctive effects of cyclosporine and macrolide in rapidly progressive cryptogenic organizing pneumonia with no prompt response to steroid. Intern Med 50: 475-479, 2011.
9. Mukae H, Kadota J, Kohno S, Matsukura S and Hara K: Increase of activated T-cells in BAL fluid of Japanese patients with bronchiolitis obliterans organizing pneumonia and chronic eosinophilic pneumonia. Chest 108: 123-128, 1995.

10. Cai M, Bonella F, Dai H, Sarria R, Guzman J and Costabel U: Macrolides inhibit cytokine production by alveolar macrophages in bronchiolitis obliterans organizing pneumonia. Immunobiology 218: 930-937, 2013.

11. Yousem SA, Lohr RH and Colby TV: Idiopathic bronchiolitis obliterans organizing pneumonia/cryptogenic organizing pneumonia with unfavorable outcome: pathologic predictors. Mod Pathol 10: 864-871, 1997.

12. Ichikawa Y, Ninomiya H, Katsuki M, Hotta M, Tanaka M and Oizumi K: Low-dose/long-term erythromycin for treatment of bronchiolitis obliterans organizing pneumonia (BOOP). Kurume Med J 40: 65-67, 1993.

13. Ishii T, Manabe A, Ebihara Y, Ueda T, Yoshino H, Mitsui T, et al: Improvement in bronchiolitis obliterans organizing pneumonia in a child after allogeneic bone marrow transplantation by a combination of oral prednisolone and low dose erythromycin. Bone Marrow Transplant 26: 907-910, 2000.

14. Stover DE and Mangino D: Macrolides: a treatment alternative for bronchiolitis obliterans organizing pneumonia? Chest 128: 3611-3617, 2005.

15. Ianaro A, Ialenti A, Maffia P, Sautebin L, Rombolà L, Carnuccio R, et al: Anti-inflammatory activity of macrolide antibiotics. J Pharmacol Exp Ther 292: 156-163, 2000.

16. Luisi F, Gandolfi TD, Daudt AD, Sanvitto JP, Pitrez PM and Pinto LA: Anti-inflammatory effects of macrolides in childhood lung diseases. J Bras Pneumol 38: 786-796, 2012.

17. Zarogoulidis P, Papanas N, Kioumis I, Chatzaki E, Maltezos E and Zarogoulidis K: Macrolides: from in vitro anti-inflammatory and immunomodulatory properties to clinical practice in respiratory diseases. Eur J Clin Pharmacol 68: 479-503, 2012.

18. Wagner T and Burns JL: Anti-inflammatory properties of macrolides. Pediatr Infect Dis J 26: 75-76, 2007.

19. Langelot M, Cellerin L and Germaud P: Anti-inflammatory effects of macrolides: applications in lung disease. Rev Pneumol Clin 62: 215-222, 2006 (In French).

20. Davidson R and Peloquin P: Anti-inflammatory effects of the macrolides. J Otolaryngol 31 (Suppl 1): 38-40, 2002.

21. Kudoh S: Erythromycin treatment in diffuse panbronchiolitis. Curr Opin Pulm Med 4: 116-121, 1998.

22. Itkin IH and Menzel ML: The use of macrolide antibiotic substances in the treatment of asthma. J Allergy 45: 146-162, 1970

23. Sharma S, Jaffe A and Dixon G: Immunomodulatory effects of macrolide antibiotics in respiratory disease: therapeutic implications for asthma and cystic fibrosis. Paediatr Drugs 9: 107-118, 2007. 